\title{
Protein Oxidation in Active and Abstinent Alcoholics in Comparison With Normal Controls
}

\author{
Authors \\ Sandhya $\mathbf{C V}^{1}$, Vidhukumar $\mathrm{K}^{2}$, Sreekumari RG $^{3}$ \\ ${ }^{1}$ Associate Professor of Biochemistry, Government Medical College, Thiruvananthapuram, Kerala, India \\ Email:drsandhyacv@gmail.com \\ ${ }^{2}$ Additional Professor of Psychiatry, Government Medical College, Thiruvananthapuram, Kerala, India \\ Email: kumarv68@gmail.com \\ ${ }^{3}$ Assistant Professor of Biochemistry, Government Medical College, Thiruvananthapuram, Kerala, India
}

\begin{abstract}
Background-Though there is much evidence in support of the hypothesis that acute or chronic alcohol toxicity is mediated primarily via the generation of damaging free radical species, only very few studies had analysed the effect of alcohol withdrawal on protein oxidation and antioxidant status in alcoholics. Also studies concentrating on alcoholics without any clinical or biochemical evidence of liver disease and eliminating other confounders such as smoking are less. Hence the present study aimed to measure carbonyl protein as an index of oxidative damage to proteins in alcoholics without liver disease and determine whether it is reversible on abstinence from alcohol.

Methods- The study was conducted in patients with alcohol dependence attending the de-addiction facility in a tertiary care hospital in south India. The study group comprised of 30 male alcoholics in the age group 20-60 years who exhibited no clinical or biochemical evidence of severe liver disease. Blood sample collection was done in the study group immediately after admission and after one month of reported abstinence from alcohol (Groups 1\&2, n=30 each). The control group (Group 3, n=30) included healthy male subjects in the same age group as alcoholics with no or occasional alcohol consumption only. Serum Protein carbonyl (PC) levels and plasma Vitamin C levels were estimated in the study and control groups..The confounding effect of smoking on the parameters of oxidative stress was eliminated by appropriate statistical methods and estimated marginal means calculated.

Results-PC levels were found to decrease significantly on abstinence from alcohol. Both active alcoholics and abstinent alcoholics show significantly elevated PC levels compared to controls even after eliminating the effect of smoking. Serum Vitamin C levels were found to be lower in both the alcoholic subgroups compared to non-alcoholic controls, but there was no significant elevation of levels on abstinence from alcohol.

Conclusion-Oxidative damage to proteins is increased in alcoholics without liver disease which comes down on abstinence. Antioxidant supplementation may be essential during the withdrawal phase to reverse this damage.

Keywords- Protein carbonyls, oxidative damage, abstinent alcoholics, alcohol withdrawal, protein oxidation, vitamin $C$.
\end{abstract}




\section{INTRODUCTION}

The occurrence of oxidative stress and Reactive Oxygen Species in the pathogenesis of alcoholic liver disease (ALD) is proven by many studies ${ }^{(1),(2)}$. Some of these studies had also evaluated the therapeutic effects of various antioxidant compounds in ALD ${ }^{(3)}$. However, later works had proven that oxidative damage due to Reactive Oxygen Species (ROS) is also present in nonalcoholic fatty liver disease and is not exclusive to ALD ${ }^{(4),(5)}$. Hence, there arises a need to examine whether the observed increase in indices of oxidative stress in ALD is actually related to alcoholism per se or to liver disease. There is also a need to find the relationship between oxidative damage and alcohol withdrawal state in a previously addicted individual.

Lipid peroxidation has been widely studied to assess oxidative stress in alcoholics along with various antioxidant enzymes ${ }^{(6)}$. In addition to lipids, other biomolecules such as proteins can also be affected by oxidative damage due to excessive ROS production. Compared to the abundance of studies on lipid peroxidation and ethanol,data available on protein oxidation after alcohol ingestion is limited. Translational research has revealed mechanisms that involve protein adducts of acetaldehyde formed from ethanol which activate profibrogenic pathways in hepatic stellate cells leading to ALD. ${ }^{(7),(8)}$ Hence it is essential to evaluate protein oxidation in relation to alcohol and analyse the changes upon abstinence.

\section{Proteincarbonyls}

Introduction of carbonyl groups into amino acid residues of proteins is a hallmark of oxidative modification by Reactive Oxygen Species. Where ever free radicals are generated, enzymes and structural proteins can be attacked by them and as a consequence, oxidative modification of proteins may occur. Metal catalysed oxidation is the most important primary mechanism for oxidative modification of proteins. Carbonyl groups (aldehydes and ketones) are added mainly to aminoacid residues such as lysine, arginine, proline and histidine. Assay of carbonyl groups provides a convenient technique for detecting and quantifying oxidative modification of proteins by ROS ${ }^{(54)}$. Its measurement also does not require expensive equipment.

Grattagliano was one of the pioneers to study protein oxidation in relation to ethanol. ${ }^{(9)} \mathrm{He}$ showed an increase in plasma \& RBC levels of carbonyl protein which was consistent with increased occurrence of protein oxidation in alcoholic liver disease, compared to non-alcoholic liver disease and normal controls. However, carbonyl protein levels did not correlate with the degree of liver injury and he concluded that protein oxidation in alcoholics does not seem to result directly from ethanol. Animal studies using rat models of ethanol toxicity also showed the same results. Bailey et al in 2001 demonstrated that chronic ethanol feeding to rats significantly increases both cytosolic and mitochondrial concentration of protein carbonyls in the liver by $30 \%$ and $60 \%$ respectively ${ }^{(10)}$. In another study, mitochondrial concentration of protein carbonyls correlated with the occurrence of alcohol induced myocardial fibrosis in experimental rats and was elevated compared to pair fed rats on normal diet (11). Yet another significant study showed that oxidative modification of low density lipoproteins (LDL) by free radicals was the reason for increased cardiovascular risk in chronic alcoholic patients by stimulating atherogenesis ${ }^{(12)}$. A major drawback of most of these studies is that they haven't taken into consideration other significant behaviours such as smoking in alcoholics which may exert a confounding effect on oxidative stress parameters.

The antioxidants that have been studied in relation to alcoholism include mostly enzymatic ones such as Glutathione peroxidase, Superoxide dismutase etc. Both thermodynamically and kinetically, ascorbate can be considered to be an excellent antioxidant and a valuable marker of oxidative stress ${ }^{(13)}$ The role of alcohol in Vitamin C status is complex and probably involves behavioural as well as metabolic influences. Alcohol consumption may reduce the intake and bioavailability of the vitamin 
and increase both the daily requirement and urinary excretion. The daily requirement is increased due to increased free radical activity associated with alcoholism $^{(14)}$.

Thus, though there is much evidence in support of the hypothesis that acute or chronic alcohol toxicity is mediated primarily via the generation of damaging free radical species, only very few studies had analysed the effect of alcohol withdrawal upon protein oxidation and antioxidant status in alcoholics. Also studies concentrating on alcoholics without any clinical or biochemical evidence of liver disease and eliminating other confounders such as smoking are less. Hence the present study aimed to measure the oxidative damage to proteins using carbonyl protein as the index in alcoholics without liver disease and determine whether it is reversible on abstinence from alcohol. It also evaluated the antioxidant status by measuring vitamin $\mathrm{C}$ levels in blood in the same group.

\section{MATERIALS AND METHODS}

The study was conducted in patients with alcohol dependence attending the de-addiction facility under the department of Psychiatry, in a Government Medical College. The study group comprised of 30 male alcoholics in the age group 20-60 years who exhibited no clinical or biochemical evidence of severe liver disease. Only those patients with a valid diagnosis of alcohol dependence as per the DSM$\mathrm{IV}^{\mathrm{TR}}$ criteria for more than 5 years were included in the study. Informed consent was obtained from each subject included in the study and the study protocol was approved by the institutional ethics committee. A detailed standardised interview aimed especially at drinking history and tobacco and drug consumption was performed on all patients and data collected. Blood sample collection was done in the study group immediately after admission and after one month of reported abstinence from alcohol. Thus the study group included two subgroups as follows: Group $1(n=30)$ included active alcoholic patients who had consumed alcohol within the past 24 hours. Group $2(n=30)$-Abstinent alcoholics include those patients who underwent dead diction therapy and are abstinent at least for one month. The control group (Group 3, n=30) included healthy male subjects in the same age group as alcoholics with no or occasional alcohol consumption only $(<3$ times a month).

The mean daily alcohol consumption, duration of alcoholism etc. were assessed with the help of a standard questionnaire. Baseline investigations, renal and liver functions tests were done on all patients and those alcoholics with alteration in liver function parameters were excluded. Also patients with all major medical illnesses like diabetes, hypertension, coronary artery disease, kidney disease and surgical illnesses were excluded from the study as they can possibly alter the oxidative stress parameters. Venous blood was collected at the time of admission and after one month of standard deaddiction therapy. Serum was used for Protein carbonyl (PC) estimation and other base line investigations and plasma for Vitamin $\mathrm{C}$ estimation. For Vitamin $\mathrm{C}$ estimation, the samples were stabilised by adding 5\% meta-phosphoric acid immediately after separation. Protein carbonyl estimation in serum was done with the method of Reznick \& Packer ${ }^{(15)}$. This method is based on the spectrophotometric detection of the reaction of 2,4dinitrophenyl hydrazine with protein carbonyls to form protein hydrazones. Vitamin $\mathrm{C}$ is estimated using the reaction with DTCS reagent ${ }^{(16)}$. All the chemicals used for reagent preparation were of analytical grade and only double distilled deionised water was used. The readings were taken in a Systronics UV-visible spectrophotometer.

Results are expressed as mean \pm standard deviation. Comparison of mean values of numerical variables between the three different study groups were done using ANOVA and Mann- Whitney \& rank sum test for normally distributed and not normally distributed data respectively, and $\mathrm{P}$ value less than 0.05 considered significant. Comparison of nominal variable (smoking) was done using Chi-square tests. Since smoking was found to be a confounding variable which differed significantly among study groups, univariate analysis of variance with smoking as covariate was done for the different 
parameters (ANCOVA), and estimated marginal means calculated eliminating the effect of smoking. These means were then compared between groups and F-value and significance calculated. Post hoc tests were also done after ANOVA has given a significant ' $F$ ' value for pair-wise comparisons between each of the two different study groups taken as a pair and significance analysed. Spearman's rho rank correlation was done to analyse the association between different study variables since some of the variables were not normally distributed.

\section{RESULTS}

Since it is well known that smoking increases free radical mediated cellular injury ${ }^{(17)}$, it is an important confounding factor affecting our study. Taking the study subjects as a whole, $42 \%$ were non-smokers and 58\% smokers. Univariate analysis of variance for PC between groups with smoking as covariate showed that, in addition to the alcohol consumption status of the individual, smoking also affects PC levels significantly. The confounding effect of smoking on the parameters of oxidative stress was eliminated by appropriate statistical methods and estimated marginal means calculated. The adjusted mean values of protein carbonyls obtained after making allowances for the smoking status of the individual in the three different study groups are given in Table 1. Post hoc tests and pairwise comparisons among the estimated marginal means in the three groups showed that there was significant difference in mean PC values among active and abstinent alcoholic groups and both active alcoholics and abstinent alcoholics show significantly elevated PC levels compared to controls. The adjusted mean values of Vitamin C obtained after eliminating the confounding effect of smoking are given in Table 2. Vitamin C levels were found to be significantly lower both in active and abstinent alcoholics compared to the control group, but there was no significant difference between plasma levels among the alcoholic subgroups. Analysis of correlation of carbonyl protein, with the other study parameters showed a significant negative correlation with vitamin $\mathrm{C}$ levels. (Spearman's rho r value 0.323 , p value$<0.05)$ There were no significant correlations for both PC and vitamin $\mathrm{C}$ with duration of alcohol dependence and average daily intake of alcohol.

Figure 1: Boxplot showing values of PCs in different groups separately for smokers and nonsmokers (Group1-active alcoholics, Group2abstinent alcoholics, Group3-controls)

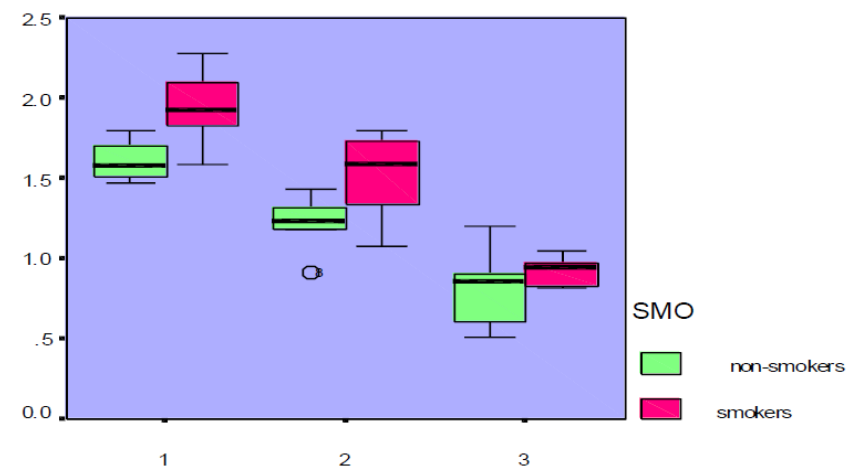

Table 1: Adjusted Mean values of serum PCs (nanomoles/mg of protein) excluding the effect of smoking

\begin{tabular}{|l|l|l|l|}
\hline Group & $\begin{array}{l}\text { Mean and95\% } \\
\text { Confidence } \\
\text { Interval }\end{array}$ & $\begin{array}{l}\mathrm{F} \\
\text { value }\end{array}$ & $\begin{array}{l}\mathrm{P} \\
\text { value }\end{array}$ \\
\hline $\begin{array}{l}\text { Active } \\
\text { alcoholics }\end{array}$ & $1.82(1.70-1.93)$ & 59.46 & $<0.001$ \\
\cline { 1 - 2 } $\begin{array}{l}\text { Abstinent } \\
\text { alcoholics }\end{array}$ & $1.39(1.28-1.50)$ & & \\
\hline Controls & $0.91(0.79-1.03)$ & & \\
\hline
\end{tabular}

Table 2: Adjusted Mean values of serum Vitamin C $(\mathrm{mg} / \mathrm{dL})$ excluding the effect of smoking

\begin{tabular}{|c|l|l|l|}
\hline Group & $\begin{array}{l}\text { Mean and 95\% } \\
\text { Confidence } \\
\text { Interval }\end{array}$ & $\begin{array}{l}\mathrm{F} \\
\text { value }\end{array}$ & $\begin{array}{l}\mathrm{P} \\
\text { value }\end{array}$ \\
\hline $\begin{array}{c}\text { Active } \\
\text { alcoholics }\end{array}$ & $1.02(0.86-1.17)$ & 5.38 & $<0.01$ \\
\hline $\begin{array}{c}\text { Abstinent } \\
\text { alcoholics }\end{array}$ & $1.02(0.86-1.17)$ & & \\
\hline Controls & $1.35(1.19-1.51)$ & \\
\hline
\end{tabular}


Figure 2: Correlation of PC with Vitamin C

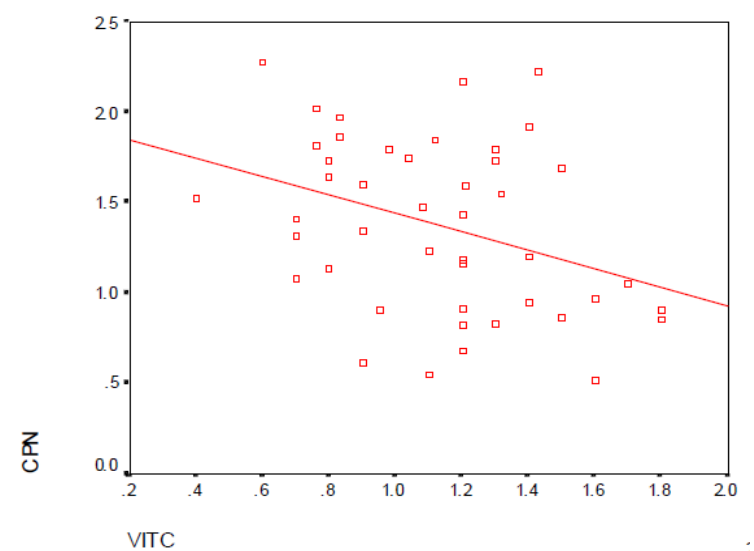

\section{DISCUSSION}

Analysis of mean values showed significantly higher levels of carbonyl protein in both active and abstinent alcoholics compared to normal controls. Also pair wise comparisons showed significant mean difference in PC levels before and after abstinence. These findings strongly suggest that alcohol induces oxidative damage to proteins which can be decreased by abstinence. In order to assess the antioxidant status, plasma Vitamin $\mathrm{C}$ levels were measured representing the non-enzymatic antioxidant group. Normal plasma concentration of vitamin $\mathrm{C}$ is between $0.4-1.5 \mathrm{mg} / \mathrm{dL}$ and values lower than $0.2 \mathrm{mg} / \mathrm{dL}$ are considered deficient. We got mean plasma concentration of $1.35+0.08$ $\mathrm{mg} / \mathrm{dL}$ in normal healthy male controls, which agree with the normal values given. All the above findings suggest lower antioxidant protection and the presence of oxidative stress in alcoholism.

Mutlu Turkoglu et al of University of Istanbul , Turkey (18) employed the same method for estimation and got results comparable to the present study. They got mean values of 1.8 nanomoles $/ \mathrm{mg}$ protein in alcoholics and 1.1 nanomoles/mg protein in the control group. Our mean values in alcoholics are similar, even though that in the control group is slightly lower. A similar study on oxidative damage to plasma proteins in patients with chronic alcohol dependence analysing the effect of smoking got significantly increased protein carbonyls in chronic alcoholics compared with smoker and non-smoker subgroups of the controls ${ }^{(19)}$. As per another study there was only a slight elevation of $20 \%$ of PC levels in alcoholics compared to normal controls ${ }^{(20)}$.
Their mean values also differ significantly from the current study as well as most of the other studies. These differences may be due to the fact that they employed a different method for estimation of PCs. Another study on rats showed a $25 \%$ increase in cytosolic and $60 \%$ increase in mitochondrial protein carbonyls in the liver as a result of chronic ethanol feeding (10). This data demonstrates that mitochondrial proteins are more vulnerable to oxidative stress after chronic ethanol consumption. Therefore it is likely that ethanol related increases in ROS might have the effect of inactivating mitochondrial proteins and this would diminish mitochondrial function and ultimately lead to the dysfunction of mitochondria in the chronic alcohol abuser.

Lower levels of Vitamin C, alpha -tocopherol and carotenoids were observed in alcohol dependent patients compared to controls and also Vitamin C levels further decreased on de-addiction therapy without supplementation of vitamins ${ }^{(14)}$. Our data also confirm this finding. Cigarette smoking is associated with decreased plasma Vitamin C levels. Smoking directly lowers the plasma Vitamin C levels by mechanisms which do not depend on dietary intake ${ }^{(21)}$. Dietary history was not taken from the study subjects; but history of drug intake showed that none of the subjects were receiving Vitamin C supplementation. Even though the alcoholic groups showed lesser levels compared to controls, they didn't fall into the deficient category. Also we can observe that even after abstinence from alcohol, the Vitamin $\mathrm{C}$ levels did not show any significant increase. This suggests the importance of supplementation of Vitamin $\mathrm{C}$ in alcoholics on dead diction therapy.

\section{CONCLUSION}

Alcohol leads to increased oxidative stress and protein oxidation which may be one of the mechanisms by which it causes cell injury. This damage is reversible on abstinence in patients without liver disease. Antioxidant supplementation may be essential during the withdrawal phase to reverse this damage. 


\section{CONFLICT OF INTEREST- None}

\section{REFERENCES}

1. Albano E. Alcohol, oxidative stress and free radical damage. Proc Nutr Soc. 2006;65(3):278-90.

2. $\mathrm{Wu} \mathrm{D}$, Cederbaum AI. Oxidative stress and alcoholic liver disease. Seminars in Liver Disease. 2009. p. 141-54.

3. Kasdallah-Grissa A, Mornagui B, Aouani E, Hammami M, Gharbi N, Kamoun A, et al. Protective effect of resveratrol on ethanolinduced lipid peroxidation in rats. Alcohol Alcohol. 2006;41(3):236-9.

4. Perlemuter G, Davit-Spraul A, Cosson C, Conti M, Bigorgne A, Paradis V, et al. Increase in liver antioxidant enzyme activities in non-alcoholic fatty liver disease. Liver Int. 2005;25(5):946-53.

5. Polimeni L, Del Ben M, Baratta F, Perri L, Albanese F, Pastori D, et al. Oxidative stress: New insights on the association of non-alcoholic fatty liver disease and atherosclerosis. World J Hepatol. 2015;7(10):1325-36.

6. Ucar G, Demir B, Ulug B. Lipid peroxidation and antioxidant enzyme activities in erythrocytes of type I and II alcoholics. Cell Biochem Funct. 2005;23(1):29-37.

7. Ceni E, Mello T, Galli A. Pathogenesis of alcoholic liver disease: Role of oxidative metabolism. World J Gastroenterol. 2014;20(47):17756-72.

8. Setshedi M, Wands JR, de la Monte SM. Acetaldehyde adducts in alcoholic liver disease. Oxid Med Cell Longev. 2010;3(3):178-85.

9. Grattagliano I, Vendemiale G, Sabbà C, Buonamico P, Altomare E. Oxidation of circulating proteins in alcoholics: Role of acetaldehyde and xanthine oxidase. J Hepatol. 1996;25(1):28-36.

10. Bailey SM, Patel VB, Young T a, Asayama $\mathrm{K}$, Cunningham CC. Chronic ethanol consumption

alters

the

glutathione/glutathione peroxidase-1 system and protein oxidation status in rat liver. Alcohol Clin Exp Res. 2001;25(5):726-33.

11. Vendemiale G, Grattagliano I, Altomare E, Serviddio G, Portincasa P, Prigigallo F, et al. Mitochondrial oxidative damage and myocardial fibrosis in rats chronically intoxicated with moderate doses of ethanol. Toxicol Lett. 2001;123(2-3):209-16.

12. Zima T, Fialov L, Mestek O, Janebov M, Crkovsk J, Malbohan I, et al. Oxidative stress, metabolism of ethanol and alcoholrelated diseases. J Biomed Sci. 2001;8(1):59-70.

13. Buettner GR, Jurkiewicz BA. Ascorbate free radical as a marker of oxidative stress: An EPR study. Free Radic Biol Med. 1993;14(1):49-55.

14. Gueguen S, Pirollet P, Leroy P, Guilland J, Arnaud J. Changes in Serum Retinol, alphaTocopherol, Vitamin C, Carotenoids, Zinc and Selenium after Micronutrient Supplementation during Alcohol Rehabilitation. J Amer Coll Nutr. 2003;22(4):303-10.

15. Reznick AZ, Packer L. Oxidative damage to proteins: Spectrophotometric method for carbonyl assay. Methods Enzymol. 1994;233:357-63.

16. Oliveira GL, Lippi G, Banfi G, Church S, Cornes M, et al. Tietz Fundamentals of Clinical Chemistry. Clin Chem Lab Med. 2007;53(3):357-70.

17. Carnevali S, Petruzzelli S, Longoni B, Vanacore R, Barale R, Cipollini M, et al. Cigarette smoke extract induces oxidative stress and apoptosis in human lung fibroblasts. Am J Physiol Lung Cell Mol Physiol. 2003;284(6):L955-63.

18. Mutlu-Türkoğlu U, Doğru-Abbasoğlu S, Aykaç-Toker G, Mirsal H, Beyazyürek M, Uysal M. Increased lipid and protein oxidation and DNA damage in patients with chronic alcoholism. J Lab Clin Med. 2000;136(4):287-91. 
19. Kapaki E, Liappas I, Lyras L, Paraskevas GP, Mamali I, Theotoka I, et al. Oxidative damage to plasma proteins in patients with chronic alcohol dependence: the effect of smoking. In Vivo. 2007;21(3):523-8.

20. Dupont I, Bodénez P, Berthou F, Simon B, Bardou LG, Lucas D. Cytochrome P-450 2E1 activity and oxidative stress in alcoholic patients. Alcohol Alcohol. 2000;35(1):98103.

21. Lykkesfeldt J, Christen S, Wallock LM, Chang HH, Jacob RA, Ames BN. Ascorbate is depleted by smoking and repleted by moderate supplementation: A study in male smokers and nonsmokers with matched dietary antioxidant intakes. Am J Clin Nutr. 2000;71(2):530-6. 